\title{
4-MCHM sorption to and desorption from granular activated carbon and raw coal
}

T. Scott Jeter ${ }^{a^{*}}$, Emily A. Sarver ${ }^{\text {a }}$, Harold M. McNairc, Mohammad Rezaee ${ }^{\mathrm{a}}$

${ }^{a}$ Mining \& Minerals Engineering, Virginia Tech, Blacksburg, VA 24060, United States

${ }^{c}$ Chemistry Department, Virginia Tech, Blacksburg, VA 24060, United States

ABSTRACT: 4-Methylcyclohexanemethanol (4-MCHM) is a saturated higher alicyclic primary alcohol that is used in the froth flotation process for cleaning coal. In early 2014, a large spill of crude chemical (containing primarily 4-MCHM) to the Elk River near Charleston, WV contaminated the local water supply. Carbon filters at the affected water treatment facility quickly became saturated, and the contaminated water was distributed to nearby homes and businesses. Sorption of 4-MCHM to granular activated carbon (GAC) was studied in the

11 laboratory using head space (HS) analysis via gas chromatography with a flame ionization 12 detector (GC-FID). Sorption to raw coal was also investigated, since this material may be of 13 interest as a sorbent in the case of an on-site spill. As expected, sorption to both materials 14 increased with decreased particle size and with increased exposure time; although exposure time 15 proved to be much more important in the case of GAC than for coal. Under similar conditions, 16 GAC sorbed more 4-MCHM than raw coal (e.g., 84.9 vs. $63.1 \mathrm{mg} / \mathrm{g}$, respectively, for $20 \times 30$ 17 mesh particles exposed to $860 \mathrm{mg} / \mathrm{L}$ 4-MCHM solution for 24 hours). Desorption from both materials was additionally evaluated. Interestingly, desorption of 4-MCHM on a mass per mass basis was also higher for GAC than for raw coal. Overall, results indicated that GAC readily sorbs 4-MCHM but can also readily release a portion of the chemical, whereas coal sorbs somewhat less 4-MCHM but holds it tightly.

Keywords: 4-Methylcyclohexanemethanol, adsorption, desorption, granular activated carbon, GC-FID, volatilization

Crude MCHM, of which 4-MCHM is major component, is most commonly used as a frothing agent in the flotation process for cleaning fine coal (Noble et al., 2015). Just a couple of

27 years ago, the chemical was not widely known, but on the morning of January 9, 2014, roughly 2810,000 gallons of crude MCHM spilled from a storage tank sitting on the bank of the Elk River 
near Charleston, WV (Aluise, 2014). The spill resulted in contamination of the local water

30 supply, and quickly spurred dialogue - and some action - at multiple levels surrounding

31 chemical regulation, emergency response and source water protection (Manuel, 2014; Rosen et al., 2014; WVL, 2014; Scaggs et al., 2015).

4-MCHM is a saturated higher alicyclic primary alcohol with two isomers (Foreman et al., 2015). The chemical is classified as nonhazardous in terms of US transportation and storage requirements, though its material safety data sheet (MSDS) describes it as a skin, eye and respiratory irritant, and says that ingestion may cause nausea, vomiting or diarrhea (Eastman, 2011). It is near colorless and moderately volatile; and its strong odor is reminiscent of licorice and mint, and is detectable at extremely low (i.e., sub-ppb) concentrations (McGuire et al., 2014; Dietrich et al., 2015; Gallagher et al, 2015).

At the time of the Elk River spill, these few facts, and the lack of more detailed information regarding human and environmental risks, combined to cause widespread panic. The spill site is just upstream of the principal intake for a West Virginia American Water (WVAW) treatment and distribution center, which serves a population of more than 300,000 . This facility continued to distribute water for hours after first receiving contaminated water from the intake (WVAW, 2014a). On the evening of the spill, WVAW concluded that its activated carbon filters could not effectively remove the MCHM from its water (Pritt, 2014), and a "do not use" order was issued to customers (WVAW, 2014a). The order remained in effect for over a week, during which time extensive flushing of the treatment facility, and plumbing systems in many affected residences and businesses, occurred. But, continued customer complaints and concerns related to odor and possible health effects of MCHM-contaminated water led WVAW to keep flushing its facility until late February 2014 (WVAW, 2014b; Whelton, 2015); and it eventually replaced the carbon media in the affected filters in June of that year (WVAW, 2014c).

Since the Elk River spill, much has been learned about the fundamental properties of 4MCHM (Noble et al., 2015; Sain et al., 2015), analytical methods for its measurement (Dietrich et al., 2015), and its behavior in premise plumbing systems following contamination (Whelton, 2015). However, the performance of sorption media for removing the chemical from water or other environmental media has not been extensively studied yet. Here, we report on basic laboratory experiments to investigate 4-MCHM uptake and holding capacity of both granular 
60 commonly used as sorbent for dissolved organics in water treatment; while raw coal, being 61 readily available onsite where crude MCHM is often used, might be employed to respond to a 62 chemical spill (Simonović et al., 2009; Bayer et al., 2005; Cornelissen et al., 2005).

2. Experimental materials and methods

A sample of crude MCHM was donated by a chemical vendor. It consisted of primarily

67 4-MCHM (i.e., approximately $86 \%$ based on reports by others using the same chemical stock

68 (Gallagher et al., 2015)), but no effort was made to identify other compounds in the sample. It

69 was stored in a five gallon bucket initially, and then a subsample was taken after vigorously

70 stirring the contents of the bucket. The subsample was used for all experiments reported here and

71 stored in $\mathrm{BD}$ Vacutainers ${ }^{\circledR}$, kept out of sunlight and in a temperature controlled lab space.

72 GAC was obtained from CABOT Norit Americas Inc. CABOT lists this particular GAC

73 as HYDRODARCO ${ }^{\circledR}$ 3000. It is an acid washed carbon designed for water treatment

74 applications (i.e., expected to have high adsorption rate and capacity for organics) (Rivera-Utrilla

75 et al., 2011). The GAC is produced from lignite that has undergone high temperature steam

76 activation. Upon receipt, the GAC was dried (at $80^{\circ} \mathrm{C}$ for 24 hours) and sized by sieving in the

77 laboratory. Three particle size classes were collected: 12 x 16 mesh $(1.19-1.68 \mathrm{~mm}), 16$ x 20

78 mesh $(0.84-1.19 \mathrm{~mm})$ and $20 \times 30$ mesh $(0.59 \times 0.84 \mathrm{~mm})$. At this point, the GAC was stored in

79 sealed plastic bags to prevent moisture uptake and oxidation; all experiments were completed 80 within 4 weeks.

81 A raw coal sample was acquired from a mine in central WV, which was operating in the

82 Upper and Lower Cedar Grove seams. The coal was run-of-mine (i.e., unprocessed) material, 83 and was stored in a five gallon bucket for several weeks before it was crushed using laboratory 
84 jaw and roll crushers, and then dried and sized similarly to the GAC. The coal was stored in

85 sealed plastic bags to minimize oxidation; then all experiments were completed within five 86 weeks.

87 Following preparation of the GAC and coal samples, proximate analysis was performed 88 on both materials to determine their oxidizable (i.e., organic) mass fraction. Higher organic

89 fraction should presumably increase organic sorption capacity of these materials (Leboeuf and 90 Weber, 2000; Delle Site, 2001, Huang et al., 2003). The analysis was performed using a Leco

91 TGA701 Thermogravimetric Analyzer. Table 1 reports the organic percent of material in each 92 size class on a dry weight basis (i.e. after moisture and volatile removal). Results represent 93 analysis of triplicate samples. A scanning electron microscope (SEM) using a backscatter 94 electron detector (BSD) was also used to image samples of both materials (e.g., see Figures S1 95 and S2 in the supplemental information). Both the proximate and SEM analyses showed that the 96 GAC had a somewhat higher oxidizable mass fraction than the coal.

Table 1: Oxidizable content in GAC and coal samples.

\begin{tabular}{|c|c|c|}
\hline \multirow{2}{*}{ Size Class (Mesh) } & \multicolumn{2}{|c|}{$\begin{array}{c}\text { Oxidizable Content } \\
\text { (mean } \pm \text { standard deviation) }\end{array}$} \\
\cline { 2 - 3 } & GAC & Coal \\
\hline $12 \times 16$ & $80.37 \pm 1.25$ & $67.83 \pm 3.49$ \\
\hline $16 \times 20$ & $83.60 \pm 1.21$ & $70.30 \pm 4.52$ \\
\hline $20 \times 30$ & $83.67 \pm 0.80$ & $72.03 \pm 0.31$ \\
\hline
\end{tabular}


An initial experiment was carried out to observe the extent to which 4-MCHM is lost due

101 to volatilization under several conditions. Three Erlenmeyer flasks were each filled with a 100

$102 \mathrm{~mL}$ of a crude MCHM solution having a concentration of $56 \mathrm{mg} / \mathrm{L}$ (or $48 \mathrm{mg} / \mathrm{L}$ as $4-\mathrm{MCHM}$ ).

103 This solution was made by dissolving the crude MCHM in deionized (DI) water, and the

104 concentration of 4-MCHM was confirmed by gas chromatography with flame ionization detector

105 (GC-FID) using a head space (HS) method as described below. All three flasks were left

106 uncovered; the first was left on the benchtop as a control (room temperature of $23{ }^{\circ} \mathrm{C}$ ), the

107 second was heated to $50^{\circ} \mathrm{C}$ on a hot plate, and the third was vigorously stirred using a magnetic

108 stir bar at room temperature. Aliquots of $10 \mathrm{~mL}$ were taken from each flask after a period of 0 ,

10915,30 , and 60 minutes. These were collected in separate $22 \mathrm{~mL}$ vials appropriate for the HS 110 analysis.

111 Next, 4-MCHM sorption and desorption experiments were carried out with both

112 materials. Several test conditions were replicated to confirm reproducibility of results (described 113 below). For the sorption experiments, four representative 1-g subsamples were split from each of 114 the sized GAC and coal samples (i.e., 24 subsamples in total). These were placed in separate 115 flasks. To each flask, $100 \mathrm{~mL}$ of a crude MCHM solution was added such that four solution 116 concentrations were tested on each material type and size. The four test concentrations were: $117125,250,500$ and 1,000 $\mathrm{mg} / \mathrm{L}$ ( or 108, 215, 430, and $860 \mathrm{mg} / \mathrm{L}$ as 4-MCHM). All of these are 118 notably below the expected maximum solubility in DI water at room temperature (Dietrich et al., 119 2015, He et al., 2015).

120 During the sorption experiments, flasks were sealed to minimize losses due to 121 volatilization. They were agitated gently on a shaker table to promote interaction between the 
122 solution and solid surfaces. After a period of 1 and 24 hours, $10 \mathrm{~mL}$ aliquots were taken from

123 each flask. The concentration of 4-MCHM in each aliquot was then measured.

124 Desorption experiments were also carried out using 20x30 mesh GAC and raw coal.

125 Again, 1-g subsamples (two of GAC and two of coal) were split from the previously sized

126 materials. Each subsample was exposed to $100 \mathrm{~mL}$ of near-saturated crude MCHM solution. The

127 solution was made by vigorously mixing $2 \mathrm{~g}$ of crude MCHM with $1 \mathrm{~L}$ of DI water for 128 approximately 5 minutes. Following mixing, some MCHM could still be observed on the

129 solution surface, indicating that the maximum solubility achievable within the mixing time had

130 been met. The insoluble MCHM layer was carefully pipetted off of the solution surface, and then

131 the solution $(950 \mathrm{mg} / \mathrm{L}$ 4-MCHM) was transferred into clean flasks with $\mathrm{GAC}$ and coal 132 subsamples. The flasks were sealed and gently agitated, for either 1 or 24 hours. Then, each 133 subsample was rinsed 20 successive times with $30 \mathrm{~mL}$ of DI water in each rinse. Rinsing was 134 done via a careful pipette-and-refill protocol: the solution in the flask was removed using a 135 pipette to avoid removal of solids, and then fresh rinse water was added to the flask and gently 136 agitated for 5 minutes. On rinses 1, 2, 3, 5, 10, 15 and 20, a $10 \mathrm{~mL}$ aliquot of the rinse solution 137 was collected and the 4-MCHM concentration was measured in each of these. 2.3. GC Analysis

141 was used with an FID. HS analysis involves measurement of the gaseous portion of 4-MCHM 142 that diffuses into the head space in a capped vial. The GC method details are summarized in 143 Table S1 for quick reference, and a brief description follows. 
Vials containing samples for 4-MCHM quantitation were heated to $80^{\circ} \mathrm{C}$ and agitated for

$14630 \mathrm{~min}$ by an HS2000 auto sampler. The sampler made injections with $0.2 \mathrm{~mL}$ head space into a

147 Thermo Scientific Finnigan TRACE GC Ultra GC, with a DB-624 column (dimensions in Table

$148 \mathrm{~S} 1$ in SI). A temperature program was used to elute the MCHM in a timely manner by increasing

149 the oven temperature from an initial $100{ }^{\circ} \mathrm{C}$ after 1 minute had passed at a rate of $20{ }^{\circ} \mathrm{C} / \mathrm{min}$ to

$150140{ }^{\circ} \mathrm{C}$, which was held for 3 minutes before the temperature was again increased at a rate of 40

$151{ }^{\circ} \mathrm{C} / \mathrm{min}$ until a final temperature of $220{ }^{\circ} \mathrm{C}$ was reached and maintained for 2 minutes. Both the

152 trans and cis isomers of 4-MCHM eluted between 5 and 6 minutes using this method. The

153 remaining 4 minutes of the method were used to ensure that minimal carryover occurred between

154 runs; and two blanks were also employed between each run for this reason. It should be noted

155 that no carryover was observed in any blanks between samples reported here.

156 The trans isomer was seen to elute before the cis isomer. A sample chromatogram from a

157 fresh MCHM solution (i.e., not having contacted either of the sorbent materials) is shown in

158 Figure S3 in SI. The trans to cis ratio in fresh solutions used in this study was consistently $1.92 \pm$

$1590.03: 1$. This is slightly higher than the ratio found in previous studies, which reported 1.75:1

160 (Dietrich et al., 2015) or 1.77:1 (Foreman et al., 2014) in solutions of crude MCHM from similar

161 chemical stocks. Notably, these studies used different analytical techniques (i.e., NMR or purge

162 and trap GC-MS, respectively) and investigated MCHM concentrations that were higher than

163 many of those investigated here.

164 A calibration curve was established using a stock solution of $100.00 \mathrm{mg} / \mathrm{L}$ crude MCHM 165 (or $86.00 \mathrm{mg} / \mathrm{L}$ as 4-MCHM) in DI water, which was diluted to concentrations of 1.25, 6.25, $16612.50,25.00$ and 50.00 (or 1.08, 5.38, 10.75, 21.50 and 43.00 as 4-MCHM). The curve exhibited 167 very good linearity above the $5.38 \mathrm{mg} / \mathrm{L}$ 4-MCHM concentration (see Figure S4 in SI), which 
168 can be taken as limit of quantitation for the HS with GC-FID method used here. At the lowest

169 concentration, 4-MCHM was detectable but the FID response was lower than predicted by the

170 linear calibration curve.

At the outset, it is important to note that the experimental methods and GC-FID with HS

173 analysis were observed yield reproducible results. Reproducibility was implied by running

174 random replicate tests (see Tables S2 and S3 in SI) as well as by analyzing replicate standard 175 solutions on several occasions. Standards solutions with target 4-MCHM concentrations of $17610.75,43.00$ and $86.00 \mathrm{mg} / \mathrm{L}$ were measured to be $10.71 \pm 0.12(\mathrm{n}=5), 43.26 \pm 0.85(\mathrm{n}=6)$ and $17785.66(\mathrm{n}=2$, values of 88.03 and 83.28$) \mathrm{mg} / \mathrm{L}$, respectively. For the volatilization experiment, 178 the initial test solution concentration was also effectively measured in triplicate (i.e., the $t=0$ 179 samples for the control, heated and stirred tests). The target 4-MCHM concentration of that 180 solution was $48.16 \mathrm{mg} / \mathrm{L}$, and the measured test solutions were determined to be $48.27 \pm 0.11$ $181 \mathrm{mg} / \mathrm{L}$.

\subsection{Volatilization of 4-MCHM}

The experiment to investigate volatilization of 4-MCHM from aqueous solution

185 confirmed that the dissolved chemical does not readily come out of solution under stagnant 186 conditions at room temperature (Table S4 in SI). In the control test, the 4-MCHM concentration 187 after 1 hour was still $99.99 \%$ of the initial concentration. This is consistent with a very small 188 Henry's Law constant (i.e., partial pressure in atmosphere to equilibrium concentration in 189 solution) which has been estimated and reported elsewhere for 4-MCHM (Gallagher et al., 190 2015). However, in tests where the solution was stirred or heated, the 1-hour concentration was 
191 only 78.09 and $67.94 \%$ of the initial, respectively. These results illustrate the relative volatility of

192 this chemical, and are in agreement with previous studies that found that constant agitation and

193 elevated temperatures increase the rate at which volatilization occurs (Noble et al., 2015).

\subsection{4-MCHM Sorption to GAC and Coal}

Though both sorbent materials were effective, the GAC sorbed substantially more 4-

198 MCHM than the raw coal when comparing similar particle sizes and exposure times (Figures 1

199 and 2, and Figures S5 and S6 in SI). The highest uptake for both materials was observed for the 200 smallest size class ( $20 \times 30$ mesh) and longer exposure time (24 hours). Under these conditions, 201 the GAC loading was found to be $84.9 \mathrm{mg} / \mathrm{g}$ and the coal loading was $63.1 \mathrm{mg} / \mathrm{g}$; however, these 202 values likely do not represent the actual sorption capacity. From Figures 1 and 2, only the 203 sorption isotherms exhibited by the larger coal particles (i.e., 12 x 60 mesh and 16 x 20 mesh)

204 indicate the maximum achievable loading was approached (i.e., loading on a mass per mass basis 205 began to level off as the residual concentration in the test solution increased). For the $20 \times 30$ 206 mesh coal and all GAC particle sizes, the sorption isotherms appear to be nearly linear, which 207 suggests that more 4-MCHM could have been loaded to these materials by test solutions with 208 higher initial concentrations.

209 With respect to GAC, the highest loading observed here $(84.9 \mathrm{mg} / \mathrm{g})$ is double that 210 reported in a prior study (i.e., about $39.3 \mathrm{mg} / \mathrm{g}$ ) (Dietrich et al., 2015). While that study does not

211 provide the particle size range of the GAC tested, the particles were presumably larger than those 212 in the $20 \times 30$ mesh sample used here. Regarding the loading to raw coal, no prior reports were 213 found to which the results presented here could be reasonably compared. Recent studies 214 surrounding use of MCHM in coal flotation, and partitioning of the chemical to process 
215 wastewaters, have indeed shown that coal significantly sorbs 4-MCHM (He et al., 2015; Noble et

216 al., 2015). But loading values were determined on coal particles from a processing facility,

217 which would have been wetted and likely contacted with other processing chemicals prior to 218 testing.

219 The trend of increased sorption with decreased particle size is expected since surface 220 area, and thus availability of sorption sites, increases as particle diameter decreases. Increased 221 sorption with exposure time is also expected - though the time significance was seen to be 222 greater for the GAC than for the coal. To illustrate, comparing all 1 and 24 hour tests (i.e. test 223 pairs having the same particle size and initial 4-MCHM concentration), sorption to the GAC 224 increased by $26 \pm 9 \%$ but sorption to the coal only increased by $4 \pm 6 \%$. The relatively quick 225 sorption to coal is consistent with recent findings by He et al. (2015). The observed difference in 226 kinetics here may mean that key differences exist in the sorption mechanisms between the two 227 materials - with one possible explanation being that GAC has higher porosity than coal, leading 228 to increased surface diffusion and number of active sorption sites. As a result of reduced pore 229 path length in GAC, inter-particle transport by the solution may relatively fast in GAC as 230 compared to coal. These characteristics, combined with the somewhat higher organic content of 231 the GAC, may translate to a relatively lower activation energy of sorption for GAC, and thereby 232 an increased rate and amount of 4-MCHM uptake (Steinberg, 1987; Pignatello, J.J., 1990; 233 Pignatello and Xing, 1996; Adamson and Gast, 1997; Aktaş and Çeçen, 2007; Shih et al., 2009).

234 Generally speaking, sorption to either material did not appear to be strongly preferential 235 for one isomer versus the other. For the 1-hour tests with both materials and the 24 hour tests 236 with coal, the trans to cis ratios in the residual solutions were near or only slightly higher (i.e., up 237 to 2.12:1) than the 1.92:1 ratio observed in fresh solutions (see data in Tables S2 and S3 in SI). 
238 This suggests that the isomers behave similarly in terms of their interaction with organic

239 surfaces, as has been observed with other organic compounds having multiple isomers (Shih and

240 Gschwend, 2009). For the 24-hour tests with GAC, the trans to cis ratio was typically observed

241 to be slightly lower (i.e., down to 1.70:1) than the 1.92:1 ratio for fresh solutions. In two of the

242 24-hour GAC tests, the 4-MCHM was almost completely sorbed (i.e., the residual concentration

243 in the test solution was less than $12 \mathrm{mg} / \mathrm{L}$ ) and the trans to cis ratio was relatively low (i.e.,

244 1:46:1 or less). While the 4-MCHM concentration in these tests was approaching the limit of

245 quantitation, other test and standard solutions with low total 4-MCHM still exhibited ratios at or

246 near the 1:92:1. As such, these results may actually indicate that preferential isomer sorption can

247 happen under some conditions - but further research is required to explore this more thoroughly.

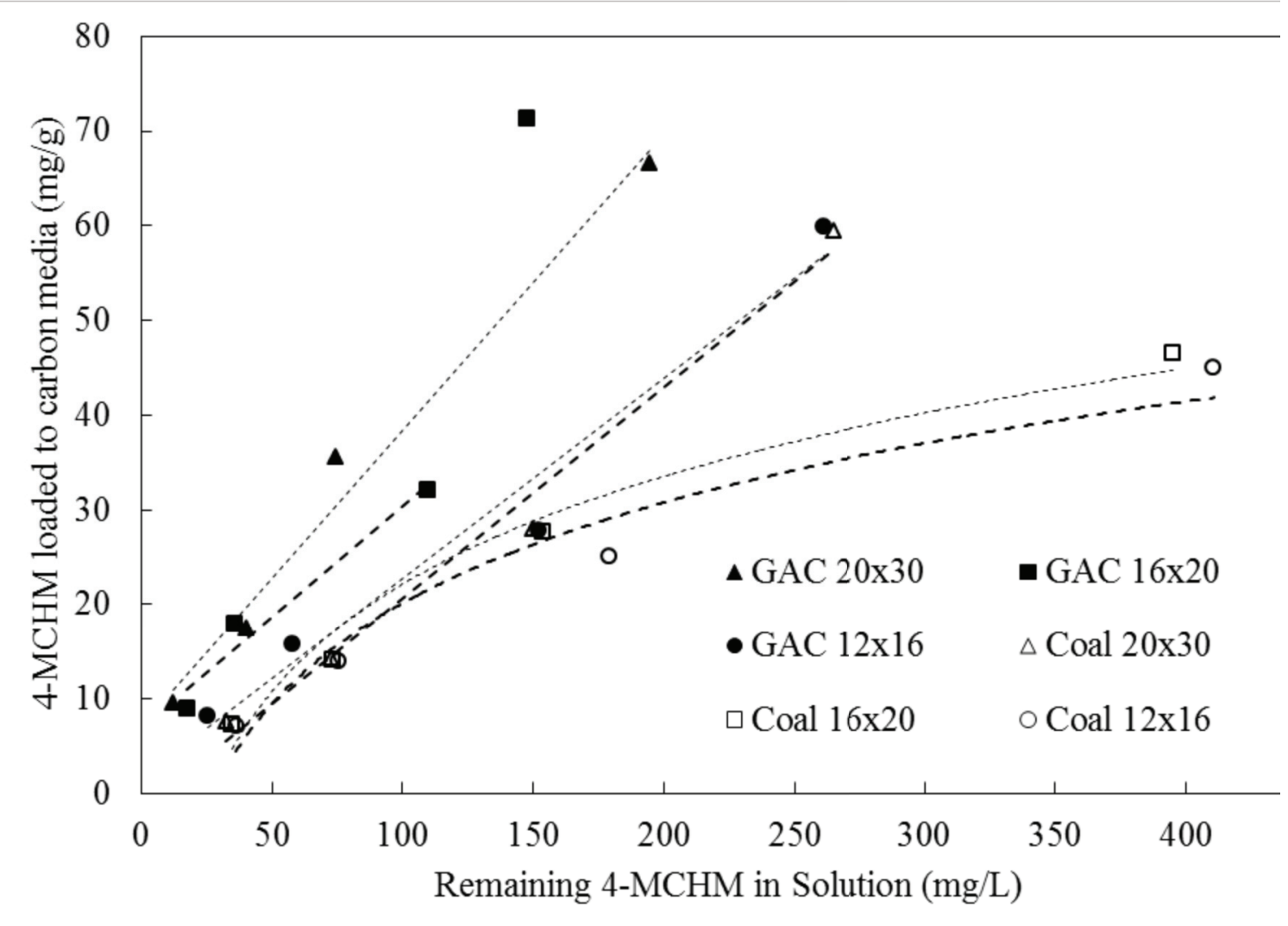

249 Figure 1. 4-MCHM loading to GAC and coal in 1-hour tests as a function of 4-MCHM 250 concentration remaining in the test solution. Note that one data point in the GAC 16 x 20 size 251 class test series appears to be an outlier and is not included in the trend-line. 


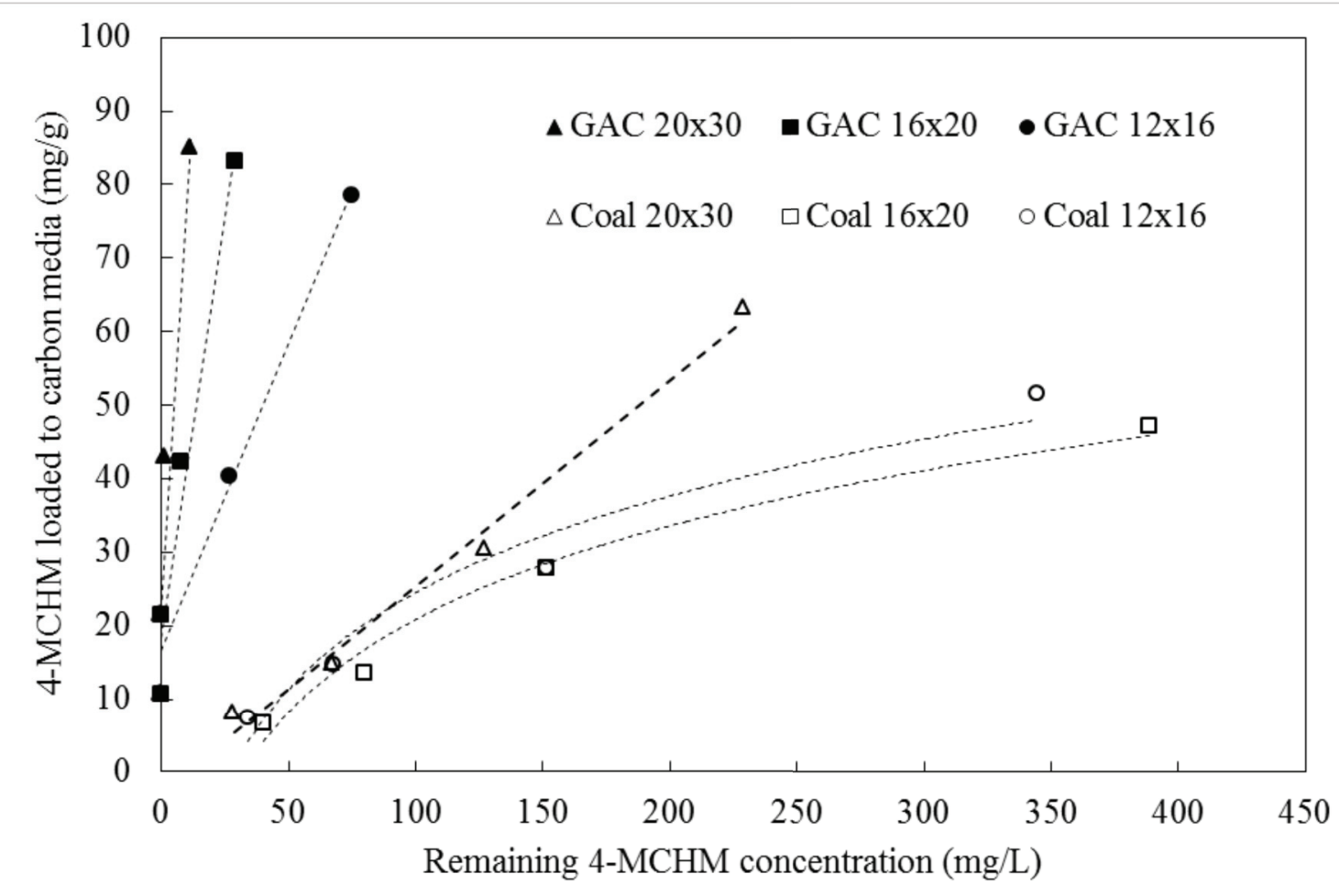

253 Figure 2. 4-MCHM loading to GAC and coal in 1-hour tests as a function of 4-MCHM 254 concentration remaining in the test solution

\subsection{Desorption}

In the desorption experiments, the effect of time was again observed. Materials exposed

258 to the crude MCHM solution for 24 hours prior to rinsing desorbed more 4-MCHM during the

259 rinsing than did materials that had only been exposed for 1 hour (Figure 3, and Table S5 and S6

260 in SI). This is probably because the materials exposed for longer were loaded with more of the

261 chemical prior to rinsing.

262 Interestingly, results from these experiments also revealed that GAC tended to desorb 263 more 4-MCHM than did coal of similar particle size. Anecdotally, this may provide some 264 explanation for the prolonged release of chemical from the WVAW filters affected by the Elk 265 River spill. Conversely, 4-MCHM is not generally found in significant concentrations in process 266 waters associated with fine coal preparation where the chemical is used as a flotation reagent 
267 (Noble et al. 2015; He et al. 2015). Taken together with the sorption results, it appears that 268 following loading and then contact with clean water the GAC and coal actually tended to hold 269 relatively similar amounts of 4-MCHM on a mass per mass basis. To explain: From Figure 3 it 270 can be determined that the 24-hour exposed coal only lost about $0.86 \mathrm{mg} / \mathrm{g}$ of 4-MCHM total -

271 i.e., about $21.5 \mathrm{mg} / \mathrm{L}$ desorbed in the first $30 \mathrm{~mL}$ of rinse water and $7.7 \mathrm{mg} / \mathrm{L}$ desorbed into the 272 second $30 \mathrm{~mL}$ of rinse water. The 24-hour exposed GAC, on the other hand, lost approximately $27312.6 \mathrm{mg} / \mathrm{g}$ of MCHM over 20 rinses (desorption estimated from the trend line for rinses where 4274 MCHM was not measured); and this material was still desorbing on the last rinse. Assuming that 275 the coal and GAC samples used in these tests had similar 4-MCHM loading as the $20 \times 30$ mesh 276 samples exposed for 24 hours in the sorption experiments (i.e., 63.1 and $84.9 \mathrm{mg} / \mathrm{g}$ ), the final 4277 MCHM held by coal and GAC would be about 62.3 and $72.2 \mathrm{mg} / \mathrm{g}$, respectively. And with 278 continued rinsing, the GAC would be expected to lose an additional $4 \mathrm{mg} / \mathrm{g}$ or so given the trend 279 for the first 20 rinses. This analysis provides further evidence that sorption mechanisms may be 280 somewhat different between the two materials.

281 Notably, the trans to cis ratio in the rinse solutions from the coal was again found to be 282 similar to the 1.92:1 ratio seen in fresh solutions - even when the total 4-MCHM concentration 283 was as low as $7 \mathrm{mg} / \mathrm{L}$. In the rinse solutions from the GAC, the ratio was also similar to that in 284 fresh solutions - except for when the 4-MCHM concentration was very low. In the test with 285 GAC that had been exposed for 1 hour, the solutions from rinse 5 had 4-MCHM of $6.55 \mathrm{mg} / \mathrm{L}$ 286 and the trans to cis ratio was $1.55: 1$; in successive rinse solutions (i.e., 10, 15 and 20), the 4287 MCHM concentration was below the limit of quantitation (though still detectable) and so a trans 288 to cis ratio was not calculated. 


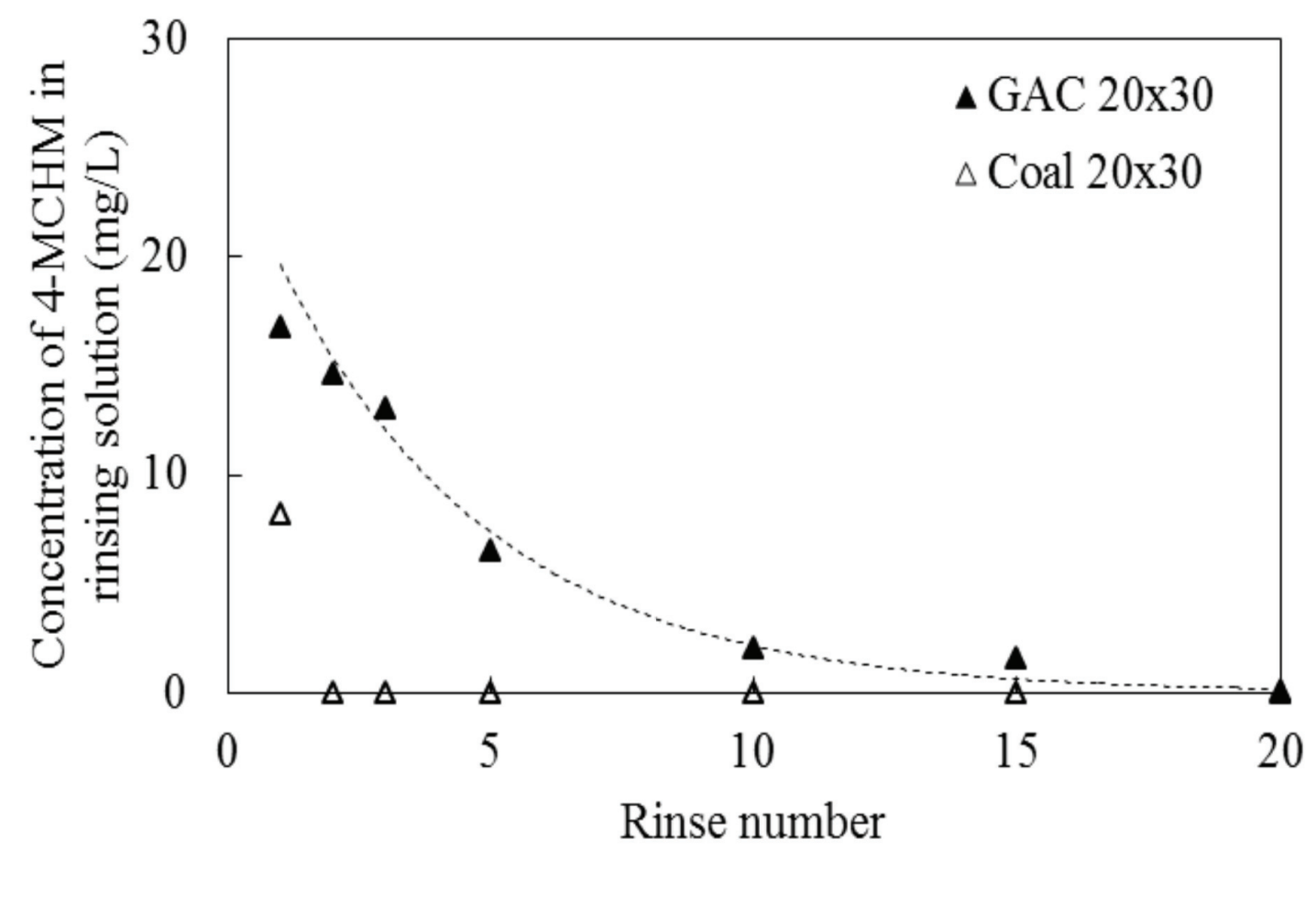

291 Figure 3: Desorption of 4-MCHM with successive rinsing in fresh DI water from $20 \times 30$ mesh 292 GAC and raw coal materials that had been exposed for 1 hour to a near-saturated crude MCHM 293 solution. Trend line is shown only for the GAC results, which were above the limit of detection 294 by the GC-FID method within all 20 rinses. For rinse numbers 10, 15 and 20, the GAC results 295 did fall below the limit of quantitation, but estimated values based on the method calibration 296 curve are include to illustrate the general data trend. For coal, only the first rinse solution showed 297 4-MCHM above the method detection limit. 


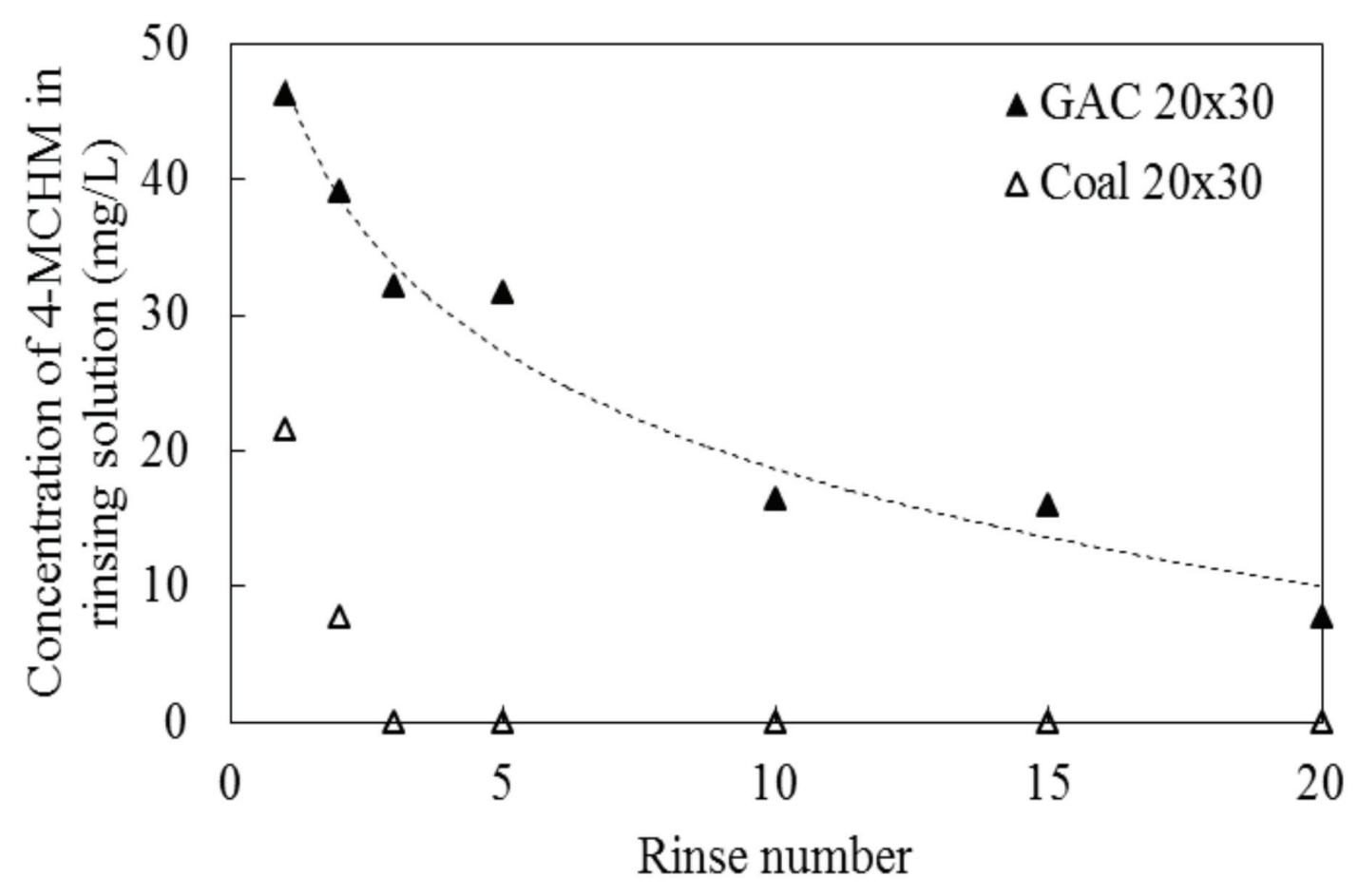

301 Figure 4: Desorption of 4-MCHM with successive rinsing in fresh DI water from $20 \times 30$ mesh 302 GAC and raw coal materials that had been exposed for 24 hours to a near-saturated crude 303 MCHM solution. Trend line is shown only for the GAC results, which were above the limits of 304 detection and quantitation by the GC-FID method within all 20 rinses. For coal, only solutions 305 from rinses 1 and 2 showed 4-MCHM above the method detection limit.

4. Conclusions Though only a limited range of experimental conditions were investigated here, some 309 important observations were made regarding sorption of 4-MCHM (from a crude MCHM 310 chemical stock) to GAC and raw coal particles. Both materials can effectively sorb the chemical,

311 but capacity and sorption kinetics vary - indicating that fundamental sorption mechanisms may 312 vary also. For similar particle sizes, GAC sorbs more 4-MCHM than does coal; and sorption 313 increases appreciably on GAC, but not coal, with increased exposure time (i.e., 24 hours versus 1 314 hour). Additionally, 4-MCHM-loaded GAC tends to desorb much more of the chemical than 315 does loaded coal. 
In general, a near 2:1 ratio between the trans and cis isomers of 4-MCHM was observed

317 in both fresh solutions and test solutions that had been contacted with GAC or coal. This

318 indicates that, for these materials and the conditions studied, sorption and desorption of 4-

319 MCHM is not preferential with respect to the isomer. Additionally, HS analysis via GC-FID

320 proved to provide reproducible results for measuring 4-MCHM concentrations in water.

321 Further research might be carried out to more definitively determine sorption

322 mechanisms and kinetics for sorbents of interest. For example, experiments with varying 4-

323 MCHM concentrations in flow through columns with sorbent media may provide additional

324 insight related to chemical uptake and holding by studying breakthrough.

Acknowledgements

The authors thank the National Science Foundation (NSF), Division of Chemical,

328 Biological, and Environmental Transport for funding part of this work under Award No.

329 1424234. Views expressed here are those of the authors and do not necessarily represent those of

330 NSF. Special thanks is also extended to Jody Smiley of the Civil and Environmental Engineering

331 Department at Virginia Tech for her invaluable help and cooperation on this project.

Adamson, A.W., Gast, a P., 1997. Physical Chemistry of Surfaces. John Whiley \& Sons: New York. SubStance 124, 192C. doi:10.1149/1.2133374. 
Aktaş, Ö., Çeçen, F., 2007. Adsorption, desorption and bioregeneration in the treatment of 2-chlorophenol with activated carbon. J. Hazard. Mater. 141, 769-777. doi:10.1016/j.jhazmat.2006.07.050

Aluise, T., 2014. Freedom Industries revises estimate for spill . West Virginia Dep. Environ. Prot. News Release. URL http://www.dhsem.wv.gov/Documents/Freedom revises estimate.pdf

Bayer, P., Heuer, E., Karl, U., Finkel, M., 2005. Economical and ecological comparison of granular activated carbon (GAC) adsorber refill strategies. Water Res. 39, 1719-1728. doi:10.1016/j.watres.2005.02.005

Cornelissen, G., Gustafsson, O., Bucheli, T.D., Jonker, M.T.O., Koelmans, A.A., Van Noort, P.C.M., 2005. Extensive sorption of organic compounds to black carbon, coal, and kerogen in sediments and soils: Mechanisms and consequences for distribution, bioaccumulation, and biodegradation. Environ. Sci. Technol. 39, 6881-6895. doi: $10.1021 / \mathrm{es} 050191 \mathrm{~b}$

Delle Site, A., 2001. Factors affecting sorption of organic compounds in natural sorbent/water systems and sorption coefficients for selected pollutants. A review. J. Phys. Chem. Ref. Data 30, 187-439. doi:10.1063/1.1347984

Dietrich, A.M., Thomas, A., Zhao, Y., Smiley, E., Shanaiah, N., Ahart, M., Charbonnet, K. a., DeYonker, N.J., Alexander, W. a., Gallagher, D.L., 2015. Partitioning, Aqueous Solubility, and Dipole Moment Data for cis - and trans -(4-Methylcyclohexyl)methanol, Principal Contaminants of the West Virginia Chemical Spill. Environ. Sci. Technol. Lett. 2, 123-127. doi:10.1021/acs.estlett.5b00061.

Eastman, 2011. Crude MCHM Safety Data Sheet. URL: http://online.wsj.com/public/resources/documents/Eastman.pdf

Foreman, W. T., Rose, D. L., Chambers, D. B., Crain, A. S., Murtagh, L. K., Thakellapalli, H., \& Wang, K. K., 2015. Determination of (4-methylcyclohexyl) methanol isomers by heated purge-and-trap GC/MS in water samples from the 2014 Elk River, West Virginia, chemical $\quad$ spill.Chemosphere, 131, 217-224. doi:10.1016/j.chemosphere.2014.11.006.

Foreman, W.T., Rose, D.L., Chambers, D.B., Crain, A.S., Murtagh, L.K., Thakellapalli, H., and Wang, K.K., 2015, Determination of (4-methylcyclohexyl)methanol isomers by heated purge-and-trap GC/MS in water samples from the 2014 Elk River, West Virginia, chemical spill: Chemosphere, v. 131, p. 217-224

Gallagher, D., Phetxumphou, K., Smiley, E., \& Dietrich, A. (2015). Tale of Two Isomers: Complexities of Human Odor Perception for cis - and trans -4-Methylcyclohexane Methanol from the Chemical Spill in West Virginia. Environ. Sci. Technol., 49(3), 1319-1327. doi:10.1021/es5049418.

He, Y.T., Noble, A., Ziemkiewicz, P., 2015. Investigation of MCHM transport mechanisms and fate: Implications for coal beneficiation. Chemosphere 127, 158-163. doi:10.1016/j.chemosphere.2015.01.054 
Huang, W., Yu, Z., Peng, P., Fu, J., 2003. Effects of organic matter heterogeneity on sorption and desorption of organic contaminants by soils and sediments. Appl. Geochemistry $18,955-972$.

Leboeuf, E.J., Weber, W.J., 2000. Macromolecular characteristics of natural organic matter. 2. Sorption and desorption behavior. Environ. Sci. Technol. 34, 3632-3640. doi:10.1021/es991104g

Manuel, J. (2014). Crisis and Emergency Risk Communication: Lessons from the Elk River Spill. Environ Health Perspect, 122 (8), A214-A219. _DOI:10.1289/ehp.122-A214

McGuire, M.J., Rosen, J., Whelton, A.J., Suffet, I.H., 2014. An unwanted licorice odor in a West Virginia water supply. J. Am. Water Works Assoc. 106, 72-82.

McNair, H.M., Miller, J.M., 2008. Basic Gas Chromatography: Second Edition, Basic Gas Chromatography: Second Edition. doi:10.1002/9780470480106

Noble, A., He, Y., \& Ziemkiewicz, P., 2015. Partitioning Behavior of 4-Methyl Cyclohexane Methanol in Two Appalachian Coal Preparation Plants. International Journal of Coal Preparation and Utilization. doi:10.1080/19392699.2015.1048335.

Pignatello, J.J., 1990. Slowly reversible sorption of aliphatic halocarbons in soils. I. Formation of residual fractions. Environ. Toxicol. Chem. 9, 1107-1115. doi:10.1002/etc.5620090901

Pignatello, J.J., Xing, B., 1996. Mechanisms of slow sorption of organic chemicals to natural particles. Environ. Sci. Technol. 30, 1-11. doi:10.1021/es940683g

Pritt, P., 2014. Cleaning chemical spill Bluef. Dly. Telegr. URL http://www.bdtonline.com/news/local_news/cleaning-chemical-spill/article_6d645638-a2805789-ae23-a936f7394c9c.html

Rivera-Utrilla, J., Sanchez-Polo, M., Gomez-Serrano, V., Alvarez, P.M., Alvim-Ferraz, M.C.M., Dias, J.M., 2011. Activated carbon modifications to enhance its water treatment applications. An overview. J. Hazard. Mater. doi:10.1016/j.jhazmat.2011.01.033

Rosen, J.S., Whelton, A.J., McGuire, M.J., Clancy, J.L., Bartrand, T., Eaton, A., Patterson, J., Dourson, M., Nance, P., Adams, C., 2014. The crude MCHM chemical spill in Charleston, W.Va. J. Am. Water Works Assoc. 106, 65-74.

Sain, A.E., Dietrich, A.M., Smiley, E., Gallagher, D.L., 2015. Assessing human exposure and odor detection during showering with crude 4-(methylcyclohexyl)methanol (MCHM) contaminated drinking water. Sci. Total Environ. 538, 298-305. doi:10.1016/j.scitotenv.2015.08.050

Scaggs, M., Sarver, E., Mendoza, L.R., 2015. The Elk River MCHM Spill : A Case Study On Managing Environmental Risks SME Min. Eng. 67(4), 48-55.

Shih, Y.H., Gschwend, P.M., 2009. Evaluating activated carbon-water sorption coefficients of organic compounds using a linear solvation energy relationship approach and sorbate chemical activities. Environ. Sci. Technol. 43, 851-857. doi:10.1021/es801663c 
Shih, Y.H., Gschwend, P.M., 2009. Evaluating activated carbon-water sorption coefficients of organic compounds using a linear solvation energy relationship approach and sorbate chemical activities. Environ. Sci. Technol. 43, 851-857. doi:10.1021/es801663c

Simonović, B.R., Arandelović, D., Jovanović, M., Kovačević, B., Pezo, L., Jovanović, A., 2009. Removal of mineral oil and wastewater pollutants using hard coal. Chem. Ind. Chem. Eng. Q. 15, 57-62. doi:10.2298/CICEQ0902057S

Steinberg, S.M., Pignatello, J.J., Sawhney, B.L., 1987. Persistence of 1,2-dibromoethane in soils: Entrapment in intraparticle micropores. Environ. Sci. Technol. 21, 1201-1208. doi:10.1021/es00165a007

West Virginia American Water (WVAW), 2014a. "Do Not Use" Order Lifted for Last Remaining Customer Area in Clendenin [Press release]. Retrieved from http://files.shareholder.com/downloads/AMERPR/3514548602x0x719617/6ecde331-c3bd44a6-b881-5183f338a5c5/FINAL LIFT NOTICE - Clendenin - 1.17.14.pdf

West Virginia American Water (WVAW), 2014b. West Virginia American Water Update: All results below lower testing level of 2 parts per billion throughout distribution system [Press $\quad$ Release]. $\quad$ Retrieved from http://files.shareholder.com/downloads/AMERPR/3514548602x0x730516/4d299ac6-eb60454a-b448-1780b4072660/WVAW_Update_-_All_results_below_2ppb.pdf

West Virginia American Water (WVAW),2014c. West Virginia American Water Carbon Filter Change-Out Project Complete [Press Release]. Retrieved from http://files.shareholder.com/downloads/AMERPR/3514548602x0x761893/976b6c49-a03c44a4-ba71-a020ee24b11f/WVAW_filter_change_out_project_complete_-_FINAL.pdf

West Virginia Legislature (WVL), 2014. An Act to Amend and Reenact §16-1-2 and §16-1$9 \mathrm{a}$ of the Code of West Virginia, 1931. Retrieved from http://www.legis.state.wv.us/bill status/bills text.cfm?billdoc=SB373\%20SUB2\%20ENR.ht $\underline{\mathrm{m} \& \mathrm{yr}=2014 \& \text { sesstype }=\mathrm{RS} \& \mathrm{i}=373}$

Whelton, A.J., McMillan, L., Connell, M., Kelley, K.M., Gill, J.P., White, K.D., Gupta, R., Dey, R., Novy, C., 2015. Residential tap water contamination following the freedom industries chemical spill: Perceptions, water quality, and health impacts. Environ. Sci. Technol. 49, 813-823. doi:10.1021/es5040969 